\title{
A Novel TDOA-Based Localization Algorithm Using Sequential Quadratic Programming
}

\author{
Xianpeng Liu ${ }^{1}$, Yi Huang ${ }^{2}$ and Jin Wang ${ }^{3}$ \\ ${ }^{\prime}$ Nantong Campus, Jiangsu City Vocational College, Nantong, Jiangsu, China \\ ${ }^{2}$ Zhangjiagang Campus, \\ Jiangsu University of Science and Technology, Zhangjiagang, Jiangsu, China \\ ${ }^{3}$ College of Information Engineering, Yangzhou University, China
}

\begin{abstract}
Linear algebraic equations based methods in the time difference of arriva (TDOA) localization are akind of excellent algorithms. In consideration of the constraints on parameters to be estimated, the first-order error in matrix and the second-order noise in vector, a sequential-quadratic-programming-based localization algorithm isproposed in this paper. An optimal solution of the linear equation model was oblained in the algorithm. Meanwhile, with the rank 1 constraint ignored, the problem yas rewritten to a series of convex functions and then a semi-definite relaxation (SDR) solution was yielded additionally. However, the solution can only be used as an initial value for other TDOA localization algorithms due to the insufficient accuracy of the SDR solution. Final localization experiments demonstrate that the proposed algorithm has a higher positioning accuracy compared with other TDOA localization algorithms based on linear model. At the same time the result can be closer to the Cramer-Rao Lower Bound, especially at the low signal to noise atio regime.
\end{abstract}

Keywords: Time Difference of Arrival (TDOA), Sequential Quadratic Programming $(S Q P)$, Semi-definite relaration $(S D R)$

\section{Introduction}

Ranging in wreless positioning system is mainly through the estimation of the distance or angle between the target node and the receiver, and then the position is calculated. Wireless ranging can be divided into received Signal Strength (RSSI) based, signal arrival angle (AOA) based and signal transmission time (TOF) based technology.

The RSSI-based techniques measure the distance between target node and receiver according to the signal strength at receiver [1].The methods are simple and easy to use, but they are/easy to be affected by multipath fading and non-line of sight in the wireless environment [2]. Furthermore, the positioning error is relatively larger, usually reaching $40 \%$ 20\%, which cannot meet the requirements of high precision positioning. The AQA-based technologies estimate the direction of transmitter to receiver by detecting phase of wireless signal at antenna array. It is less affected by SNR, but the impact of channel interference and multipath is significant [3]. Ranging technologies based on TOF are the most widely studied category [3-7], in which TDOA-based technology attaches more attention because of relatively easy achievement. In $2 \mathrm{D}$ plane, the points with a determined difference of distance to two anchor nodes (with known coordinates) lie on a hyperbolic curve. If there are three or more non-collinear anchor nodes, multiple hyperbolic curves exist, whose crossover point is the target node. Multiple equations can be obtained through these curves, and all the equations are nonlinear. Hence it is difficult to solve them. In addition, under the influence of noise, the curves can never intersect at one point. 
For decades, the TDOA localization algorithm can be divided into two types. One is based on the initial nonlinear model and estimates the target position by iterative algorithms of NLS or ML estimation, such as Taylor's series iteration (NLS) and semi-definite relaxation (SDR)-based method. This kind of algorithms requires initial values with sufficient accuracy to avoid local optimum. The other one converts nonlinear equations into linear forms via equation transformation with no error and produces approximate solutions using the least squares or weighted least squares (LS) method[4-6], which then develops into the linear-correction least square[7], total least squares[8, 9], generalized total least squares[10], constrained total least squares[1], et al. Among the algorithms mentioned above, the first kind that is based on initial models mostly depends on iteration for solution, with no guarantee for convergence and high complexity of computation. However, the result is relatively more accurate. The second kind has better linear forms, which is clear and good for analyzing, although it is not based on initial models and its optimal solution is only the suboptimal solution of initial models. It has been theoretically proved that the localization accuracy of all the algorithms above can achieve the Cramer-Rao lower bound under small error conditions.

\section{Closed Localization Algorithm and SQP Solution based Least Squares (LS)}

In the time difference of arrival (TDOA) system, the measured value is the difference of distance from the target node to each anchor node. In a general 2D localization problem, the coordinates of the target node is defined as $\hat{\mathbf{x}}=[x, y]^{T}$; the coordinates of the anchor node, $\mathbf{x}_{i}=\left[x_{i}, y_{i}\right]^{T}$; the Euclidean distance between the target and anchor node, $r_{i}=\left\|\mathbf{x}_{i}-\mathbf{x}\right\| ;$ the electromagnetic propagation velocity, c (assumed to be constant). Then the distance difference between any two coutes caused by time difference can be expressed as follows:

$$
\begin{aligned}
& r, j=c t_{i, j}=c t_{i}-c t=r_{i}-r_{j}=\left\|\mathbf{x}_{i}-\mathbf{x}\right\|-\left\|\mathbf{x}_{j}-\mathbf{x}\right\| \\
& =\sqrt{\left(x_{i}-x\right)^{2}+\left(y_{i}-y\right)^{2}}-\sqrt{\left(x_{j}-x\right)^{2}+\left(y_{j}-y\right)^{2}}, \\
& i, j=1,2,3 \ldots N
\end{aligned}
$$

In Equation (1), $\mathrm{N}$ stands (or the number of anchor nodes.

That is to say, the data that can be directly observed are $r_{i, j}$, which are $C_{N}^{2}$ in total. With no noise, Equation (2) holds.

$$
r_{i, j}=r_{i, l}+r_{l, j}, i, j, l=1,2,3 \ldots N, i \neq j \neq l
$$

If $j=1$ is used as the reference point, any $r_{i, j}$ can be calculated, with $r_{i, 1}, i=2.3 . . N$ known. Because error exists in reality, the true value is defined as $r_{i, j}^{0}$; the nolse, $n_{i, j}$. Without loss of generality, the reference anchor node is assume to be $j=1$ ;the noise vector $\mathbf{n}=\left[n_{2,1}, n_{3,1}, \ldots, n_{N, 1}\right]^{T}$; the observed distance difference vector, $\mathbf{r}=\left[r_{2,1}, r_{3,1}, \ldots, r_{N, 1}\right]^{T}$. Then the following equation is obtained:

$$
r_{i, j}=r_{i, j}^{0}+n_{i, j}
$$

In order to overcome the intrinsic shortcomings of iteration algorithms - the effectiveness of starting point and high complexity, people starts searching for an effective closed-form localization algorithm. This kind of algorithm is mainly LS-based. Because the LS solution is a closed form solution and the corresponding 
numerical methods are relatively highly developed, such as the Lanczostri diagonalizing[12] and the bi-diagonalizing in QR iteration[13] which can be easily applied to calculating solution such as DSP and so on, several effective TDOA closed localization algorithms have been developed since [4]. The typical algorithms are the spherical-interpolation [4], Chan[6], linear-correction least squares (LCLS) [7] and constrained total least squares (CTLS)[1], et al. Before discussing the SQP algorithm, the description of the linear model is given below. Because

$$
r_{i, 1}+r_{1}=r_{i}
$$

the Equation (5) can be obtained by substituting the expression of $r_{i}$ and $r_{1}$ into Equation (4) and then squaring both sides of the equation.

$$
\begin{aligned}
& \left(x_{i}-x_{1}\right)\left(x-x_{1}\right)+\left(y_{i}-y_{1}\right)\left(y-y_{1}\right)+r_{i, 1} r_{1} \\
& =\frac{1}{2}\left[\left(x_{i}-x_{1}\right)^{2}+\left(y_{i}-y\right)^{2}-r_{i, 1}{ }^{2}\right]
\end{aligned}
$$

The matrix form of Equation (5) is as follows:

$\mathbf{A \theta}=\mathbf{b} \quad(6)$

where

$$
\mathbf{A}=\left[\begin{array}{ccc}
x_{2}-x_{1} & y_{2}-y_{1} & r_{2,1} \\
x_{3}-x_{1} & y_{3}-y_{1} & r_{3,1} \\
\vdots & \vdots & \vdots \\
x_{M}-x_{1} & y_{M}-y_{1} & r_{M, 1}
\end{array}\right] \boldsymbol{\theta}=\left[\begin{array}{c}
x-x_{1} \\
y-y_{1} \\
r_{1}
\end{array}\right] \boldsymbol{b}=\frac{1}{2}\left[\begin{array}{c}
\left(x_{1}\right)^{2}+\left(y_{2}-x_{1}\right)^{2}-r_{2,1}^{2} \\
\left(x_{3}\right)^{2}+\left(y_{3}-y_{1}\right)^{2}-r_{3,1}^{2} \\
\vdots \\
\left(x_{M}\right)^{2}+\left(y_{M}-y_{1}\right)^{2}-r_{M, 1}^{2}
\end{array}\right]
$$

Equation (6) is a set of linear equations. For a general 2D localization problem, $\mathrm{N}$ $\geq 3$. Hence $\mathbf{A}$ is an over determined equation and the simplest optimal model is the following LS problem.

$$
\begin{aligned}
& \hat{\mathbf{\theta}}=\arg \min _{\boldsymbol{\theta}}(\hat{\mathbf{A} \theta-\mathbf{b}})^{T}(\mathbf{A} \boldsymbol{\theta}-\mathbf{b}) \\
& =\left(\mathbf{A}^{\mathrm{T}} \mathbf{A}\right)^{-1} \mathbf{A}^{\mathrm{T}} \mathbf{b}
\end{aligned}
$$

This is the basic LS solution, which is only suitable for systems with very low noise, and therefore it is not accurate enough. First, the LCLS algorithm has been proposed because of the constraints on parameters to be estimated. Second, because A has errors and moreover one of its has the errors rather than all, the generalized total least squares and CTLS algorithm have been developed. Third, it is widely known that to ensure the accuracy of TDOA localization based on LS requires that the error in $\mathbf{b}$ only has first order terms However, Equation (6) includes second order term of noise. Chan, LCLS and CTKS all ignore this second order term, which is only effective and can guarantee the estimation accuracy under conditions with small signal to noise ratio. When the signal to noise ratio is relatively high, the second order noise term cannot be negleeted, or the localization accuracy will be heavily impacted. Here, taking the parameter constraints, error in $\mathbf{A}$ and second order noise term into consideration, a SQR based [11] TDOA localization algorithm has been proposed, before which a SDR solution is also obtained.

First, considering the second order noise term, Equation (6) can be expressed as follows:

$$
\mathbf{A \theta}-\mathbf{b}=\Delta \mathbf{A} \boldsymbol{\theta}-\Delta \mathbf{b}=r_{1} \mathbf{n}-\mathbf{H}_{1} \mathbf{n}-\frac{1}{2} \mathbf{n} \square \mathbf{n}
$$

In Equation (8), $\mathbf{H}_{1}=-\operatorname{diag}(\mathbf{r})$ and is a diagonal matrix; $\square$ is the elements-by-elements product operation of matrix. Then, the localization problem of the model (6) is equal to the following optimizing problem: 


$$
\begin{aligned}
& \hat{\boldsymbol{\theta}}=\underset{\theta, n}{\arg \min }\|\mathbf{n}\|^{2} \\
& \text { s.t. } r_{1} \mathbf{n}-\mathbf{H}_{1} \mathbf{n}-\frac{1}{2} \mathbf{n} \square \mathbf{n}-\mathbf{A} \boldsymbol{\theta}+\mathbf{b}=0 \\
& \boldsymbol{\theta}^{T} \Sigma \boldsymbol{\theta}=0 \\
& \quad r_{1} \geq 0
\end{aligned}
$$

The constraints of the first equation of Equation (9) can be divided into N-1 equations.

$$
2 r_{1} n_{i, 1}-n_{i, 1}^{2}+2 r_{i, 1} n_{i, 1}-2 a_{i-1} \boldsymbol{\theta}+2 b_{i-1}=0, i=2,3, \ldots, N
$$

In Equation (10), $a_{i}$ and $b_{i}$ respectively stand for the row $\mathrm{i}$ of $\mathbf{A}$ and $\mathbf{b}$. Equation (10) is an indefinite quadratic constraint and can be transformed into a more regular form as follows:

$$
\begin{aligned}
& \hat{\mathbf{y}}=\underset{\mathbf{y}}{\arg \min } \mathbf{y}^{T} \mathbf{C y} \\
& \text { s.t. } \mathbf{y}^{T} \mathbf{B}_{i} \mathbf{y}=0, i=2,3, \ldots, N \\
& \mathbf{y}^{T} \mathbf{D} \mathbf{y}=0 \\
& \mathbf{y}^{T} \mathbf{E} \mathbf{y}=1 \\
& \mathbf{y}(3) \geq 0
\end{aligned}
$$

In Equation (11), $\quad \mathbf{B}_{i}=\left[\begin{array}{cc}\mathbf{0} & \mathbf{J}_{1}^{T} \\ \mathbf{J}_{1} & \mathbf{J}_{2}\end{array}\right]$, where

$$
\mathbf{J}_{1}=\left[\begin{array}{ccc}
0 & 0 & 1 \\
0 & 0 & 0 \\
0 & 0 & 0 \\
\vdots & \vdots & \vdots \\
-a_{2}(1) & -a_{2}(2) & -a_{2}(3)
\end{array}\right], \boldsymbol{J}_{2}=\left[\begin{array}{cccc}
-1 & 0 & \cdots & r_{2,1} \\
0 & 0 & \cdots & 0 \\
\vdots & \ddots & \vdots \\
r_{2,1} & 0 & \cdots & 0
\end{array}\right] .
$$

The other $\mathbf{B}$ is similar. $\mathbf{C}\left[\begin{array}{ll}\mathbf{0} & \mathbf{0} \\ \mathbf{0} & \mathbf{I}\end{array}\right], \mathbf{D}=\left[\begin{array}{ll}\Sigma & \mathbf{0} \\ \mathbf{0} & \mathbf{0}\end{array}\right], \mathbf{E}=\left[\begin{array}{ll}\mathbf{0} & \mathbf{0} \\ \mathbf{0} & 1\end{array}\right], \mathbf{y}=\left[\begin{array}{l}\boldsymbol{\theta} \\ \mathbf{n} \\ \rho\end{array}\right]$.

The dimension of the unknown parameter $\mathbf{y}$ is $\mathrm{N}-1+3+1=\mathrm{N}+3 \cdot \mathbf{y}^{T} \mathbf{E y}=1$, namely $\rho^{2}=1$, which is an additional variable used for problem regularization, and its final solution is $\mathbf{y} / \rho$. It should be noted that Equation (9) is equal to Equation (11). Through regularization, it is clearly shown that $\mathbf{B}_{i}$ and $\mathbf{D}$ are indefinite symmetric diagona matrixes. Accordingly, the correlation function is a non-convex function. Because $\mathbf{y}^{T} \mathbf{X y}=\operatorname{tr}\left\{\mathbf{X y} \mathbf{y}^{T}\right\}=\operatorname{tr}\{\mathbf{X Y}\}$, where $\operatorname{tr}\{\bullet\}$ refers to trace operation, the Equation (9) can be transformed into the optimizing problem expressed by the following equation. 


$$
\begin{aligned}
& \hat{\mathbf{y}}=\underset{\mathbf{y}}{\arg \min } \operatorname{tr}\{\mathbf{C Y}\} \\
& \text { s.t. } \operatorname{tr}\left\{\mathbf{B}_{i} \mathbf{Y}\right\}=0, i=2,3, \ldots, N-1 \\
& \quad \operatorname{tr}\{\mathbf{D Y}\}=0 \\
& \operatorname{tr}\{\mathbf{E Y}\}=1 \\
& \quad \mathbf{y}(3) \geq 0 \\
& \quad \operatorname{rank}(\mathbf{Y})=1
\end{aligned}
$$

The optimizing problem, Equation (12) is convex, neglecting the rank 1 constraint; however, the rank 1 constraint is non-convex, which complicates the problem. The solution of this kind of problem has always been the research hot spot[14-16]. SDR technology [16] is one way to solve it. The solution gained by taking out the rank 1 constraint can be regarded as suboptimal or used as the starting point for other algorithms, although it is not the optimal solution for Equation (12). Because the estimation accuracy of SDR is not enough (discussed in details in simulation part), a SQP-based algorithm is give below.

For the optimization problem Equation (9), all the constraints are equations, except the constraint $r_{1} \geq 0$. It is shown by simulation that neglecting $r_{1} \geq 0$ no influence on estimation. Hence, Equation (13) can be obtained using the lagrangian multiplier method.

$$
L\left(\mathbf{y}^{\prime}, \lambda\right)=\mathbf{n}^{T} \mathbf{n}-\lambda_{1}^{T}\left(r_{1} \mathbf{n}-\mathbf{H}_{1} \mathbf{n}-\frac{1}{2} \mathbf{n} \square \mathbf{n}-\mathbf{A} \boldsymbol{\theta}+\mathbf{b}\right)-\lambda^{\prime} \boldsymbol{\theta}^{T} \mathbf{\Sigma} \boldsymbol{\theta}
$$

In Equation (13), $\mathbf{y}^{\prime}=\left[\theta, \mathbf{n}^{T}\right]^{T}$ and $\lambda=\left[\lambda_{1}^{T}, \lambda^{\prime}\right]^{T}$ are lagrangian multiplier vectors. Using derivation operator $\nabla$, the Jacofian matrix $\mathbf{J}(0)$ can be defined as follows:

$$
\mathbf{J}\left(\mathbf{y}^{\prime}\right)^{T}=\left[\nabla c_{2}\left(\mathbf{y}^{\prime}\right), \nabla c_{3}\left(\mathbf{y}^{\prime}\right), \ldots \sigma_{c_{N}}\left(\mathbf{y}^{\prime}\right), \nabla d\left(\mathbf{y}^{\prime}\right)\right]
$$

In Equation (14), $\quad \nabla c_{j}\left(\mathbf{y}^{\prime}\right)=2 \mathbf{B}_{i}^{\prime} \mathbf{y}^{\prime}+2 \omega, d\left(\mathbf{y}^{\prime}\right)=\theta^{T} \boldsymbol{\Sigma} \theta, \nabla d\left(\mathbf{y}^{\prime}\right)=2 \mathbf{D}^{\prime} \mathbf{y}^{\prime}$, where

$$
\mathbf{B}_{i}^{\prime}=\mathbf{B}_{i}(1: N+2,1 \cdot N+2), \mathbf{D}^{\prime}=\mathbf{D}(1 \cdot N+2,1: N+2), \omega=\mathbf{B}_{i}(1: N+2, N+3) \text {. }
$$

It can be seen from the SQP algorihm[11] that for Newton iteration at Step k, Equation (15) should be satisfied.

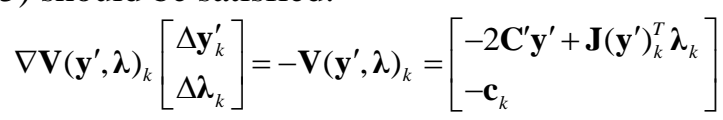

In Equation $(15) \mathbf{c}\left(\mathbf{y}^{\prime}\right)=\left[c_{2}\left(\mathbf{y}^{\prime}\right), c_{3}\left(\mathbf{y}^{\prime}\right), \ldots, c_{N}\left(\mathbf{y}^{\prime}\right)\right]^{T}, \mathbf{C}^{\prime}=\mathbf{C}(1: N+2,1: N+2)$, $\mathbf{V}\left(\mathbf{y}^{\prime}, \boldsymbol{\lambda}\right)=\left[\begin{array}{l}2 \mathbf{C}\left(\mathbf{y}-\mathbf{J}\left(\mathbf{y}^{\prime}\right)^{T} \boldsymbol{\lambda}\right. \\ \mathbf{c}(x)\end{array}\right]$, and $\nabla \mathbf{V}\left(\mathbf{y}^{\prime}, \boldsymbol{\lambda}\right)_{k}=\left[\begin{array}{cc}\nabla_{\mathbf{y}^{\prime} \mathbf{y}^{\prime}}^{2} L\left(\mathbf{y}^{\prime}, \boldsymbol{\lambda}\right)_{k} & -\mathbf{J}\left(\mathbf{y}^{\prime}\right)_{k}^{T} \\ \mathbf{J}\left(\mathbf{y}^{\prime}\right)_{k} & \mathbf{0}\end{array}\right]$.

The SQP update process is shown as below.

$$
\left[\begin{array}{l}
\mathbf{y}_{k+1}^{\prime} \\
\boldsymbol{\lambda}_{k+1}
\end{array}\right]=\left[\begin{array}{l}
\mathbf{y}_{k}^{\prime} \\
\boldsymbol{\lambda}_{k}
\end{array}\right]+\left[\begin{array}{c}
\Delta \mathbf{y}_{k}^{\prime} \\
\Delta \boldsymbol{\lambda}_{k}
\end{array}\right]
$$

The SQP process above requires a starting point close enough to the optimal point, and using the SDR solution as the starting point generally can make the process converge at the global optimum. After setting up termination criteria, the final TDOA coordinate estimation is:

$$
\hat{\mathbf{x}}=\hat{\mathbf{y}}^{\prime}(1: 2)+\mathbf{x}_{1}
$$




\section{Simulation Results}

Here five localization algorithms, least squares (LS), linear correction least squares (LCLS), constrained total least squares (CTLS), semi-definite relaxation (SDR) and sequential quadratic programming (SQP) are compared with each other, because all of them are based on the same model, Equation (6). Taking LS into account is to compare it with SDR. Since both of them can only be used to provide initial estimation for other algorithms, the compare is meaningful. The anchor nodes for simulation are distributed as follows:

$$
\begin{aligned}
& \quad \mathbf{x}_{1}=[-47,39]^{T}, \quad \mathbf{x}_{2}=[-18,-11]^{T}, \quad \mathbf{x}_{3}=[6,-10]^{T}, \quad \mathbf{x}_{4}=[26,-74]^{T}, \\
& \mathbf{x}_{5}=[61,-1]^{T}, \quad \mathbf{x}_{6}=[0,9]^{T}, \quad \mathbf{x}_{7}=[60,-43]^{T}, \quad \mathbf{x}_{8}=[-52,-47]^{T}, \quad \mathbf{x}_{9}=[-30,1]^{T}, \\
& \text { and } \mathbf{x}_{10}=[-5,17]^{T} .
\end{aligned}
$$

$\mathbf{x}_{1}$ is defined as the reference point. Except the reference point, the number of anchor nodes used in simulation $M \in\{4, \ldots, N-1\}$, and increases from $\mathbf{x}$ to $\mathbf{x}_{10}$. The absolute difference increases from 1 to 5 . For each lest configuration of each algorithm, 10,000 times Mento-Carlo simulation are run, with the mean/square error used as the evaluation index. The termination threshold value for CTLS and SQP is 0.001 , and the Sedumi package is used for SDR

It can be seen from Figure 1:

(1) The performances of SDR and LS are almost equal and inferior to other algorithms, which can however be used n initial estimation of other algorithms due to their simplicity. This study considers SDR in order to study how to take the rank 1 constraint into account in the view of convex optimization.

(2)The SQP algorithm has the pest localization performance and the result is closer to Cramer-Rao lower bound. Thereason for this is that the second order noise term and correlation of unknown parameters are considered in a unified way when SQP is used to solve op(imization problems.

(3) When there are only a few anchor nodes, or the noise is significant, the estimation performance of LCLS and CTLS is inferior to that of SQP. However, as the number of anchor nodes increases, or noise decreases, their performance is approaching that of SQP
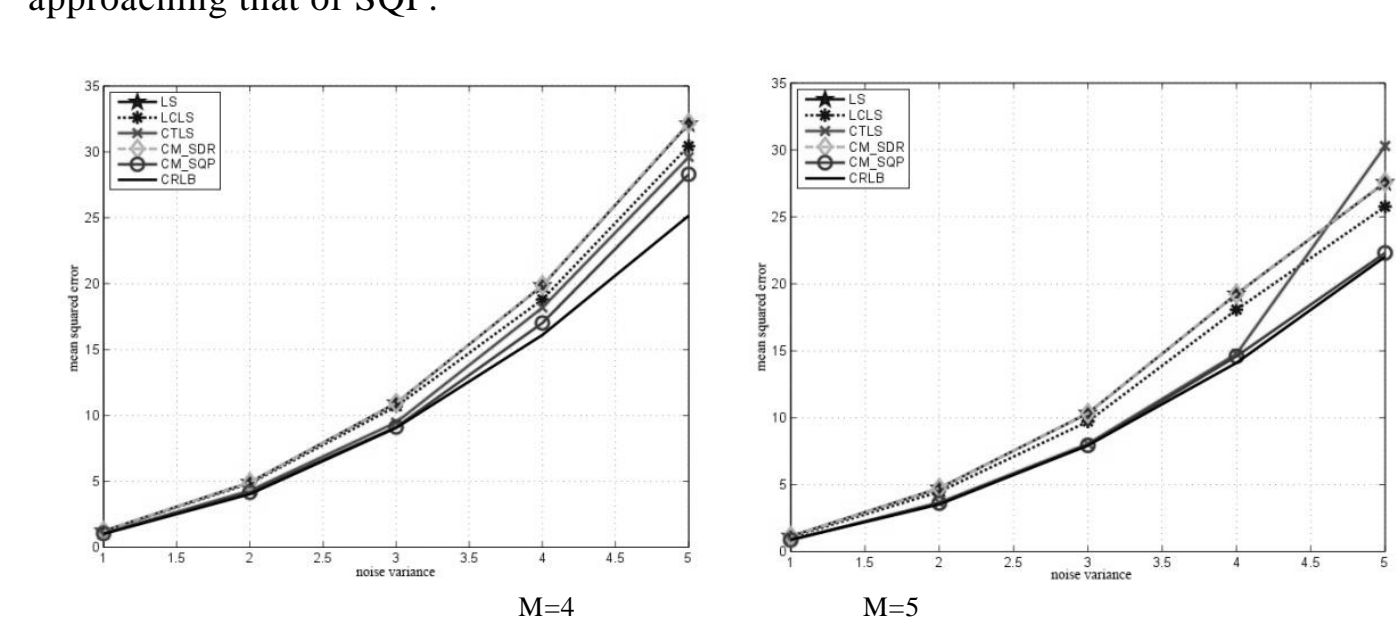

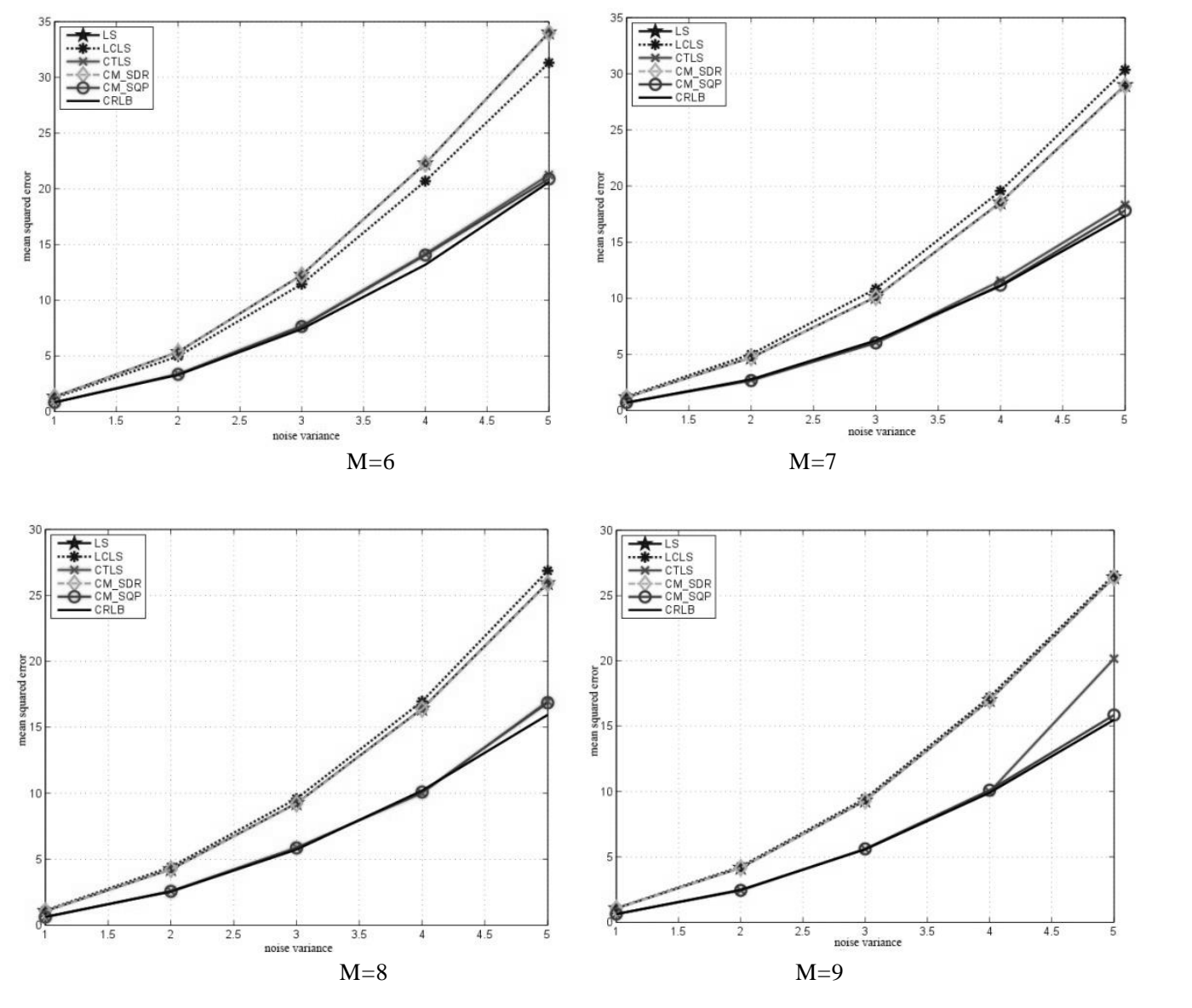

Figure 1. Localization Accuracy Compare among Five Algorithms

\section{Conclusions}

TDOA localization method has alyays been a research hot spot. For linear models of TDOA localization, a sequential quadratic programming based TDOA localization algorithm has been proposed, which takes the constraint of unknown parameters, the first order matrix error and second order noise in vectors into consideration, and produces the optimal solution of the linear model. Before the SQP process, a semi-definite relaxation solution is obtained first by transforming the problem into the convex function form and neglecting the rank, 1 constraint. Due to the accuracy insufficiency of the SDR solution, it can only be used as initial value for other algorithms. The simulation result shows the proposed SQP localization algorithm has superior localization accuracy, compared with other algorthms based on linear models, especially with low signal to noise ratio, where the result is much closer to the Cramer-Rao lower bound.

\section{Acknowledgments}

This paper is a revised and expanded version of a paper entitled "A TDOA Localization Algorithm Based on Sequential Quadratic Programming" presented at ISI 2016, August 19-20, Harbin, China. This research work is supported by the National Natural Science Foundations of China under Grant (61402234, 61402235), and by the talent project of "Green Yangzhou and golden phoenix" under contract 2013-50. 


\section{References}

[1] J.G. Hwang, K.E. Lee and J. G. Park, "An AP Selection Criteria for Enhanced Indoor Positioning using IEEE 802.11 RSSI", Measurements and AP Configuration Information. Journal of Electrical Engineering\& Technology, vol. 11, no. 2, (2016).

[2] R. Wang, S. Zheng and H. Chen, "An cooperative Localization Method Based on Taylor and Kalman Algorithms", Chinese Journal of Sensors and Actuators, vol. 27, no. 11, (2014).

[3] W. Lu, C. Liu and Q. Zhou, "TDOA Passive Location Based on Improvement of Second Cone Relaxation and Taylor-series Estimation”, Journal of Signal Process, vol. 30, no. 10, (2014).

[4] B. Xu, W.D. Qi and L. Wei, "Turbo-TSWLS: enhanced two-step weighted least squares estimator for TDOA-based localization", Electronics Letters, vol. 48, no. 25, (2012).

[5] Y. Huang, J. Benesty and G.W. Elko, "Real-time passive source localization: A practical linear-correction least-squares approach", IEEE Transactions on Speech and Audio Processing, vol. 9, no. $8,(\mathbf{2 0 0 1})$.

[6] F. Qu and X. Meng, "Source Localization Using TDOA and FDOA Measurements Based on Constrained Total Least Squares Algorithm", Journal of Electronics and Information Technology, vol. 36, no.5, (2014).

[10] Q. Lin and L. Xiao, "An adaptive accelerated proximal gradient method and its homotopy continuation for sparse optimization”, Computational Optimization and Applications, vol. 60, no. 3, (2015)

[11] I. M. Bomze, L. Grippo and L. Palagi, "Unconstrained formulation of standard quadratic optimization problems", TOP, vol. 20, no. 1, (2012).

[12] F. Zhu and P. J. Antsaklis, "Optimal control of hybrid switched systems: A brief survey", Journal of Global Optimization, vol. 25, no. 3, (2015).

[13] E. R. Van Dam and R. Sotirov, "Semidefinite programming and eigenvalue bounds for the graph partition problem", Mathematical Programming, vol. 15, no. 2, (2015).

[14] J. F. Sturm, "Using SeDuMi 1.02, a MATLAB toolbox for optimization over symmetric cones", Optimization Methods and Software, (1999).

[7] K. Yang, J. An and Z. Xu, "A Generalized Total Least-Squares^Algenthm for Hyperbolic Location. In: Wireless Communications, Networking and Mobile Computing", 2008. WiCOM '08.4th International Conference on, (2008).

[8] J. Nocedal and S. J. Wright, "Numerical Optimization", Springe, (2006).

[9] G. H. Golub, F. C., V. Loan, "Matrix Computation", JWU Press, (2013).
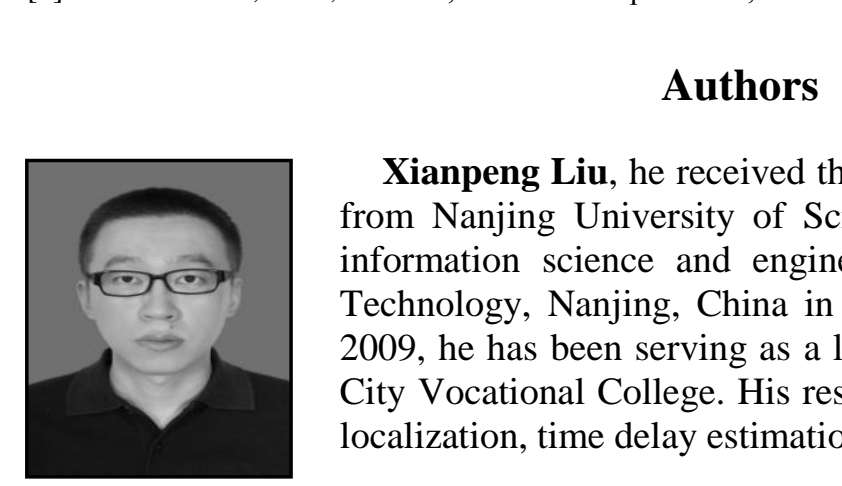

Xiampeng Liu, he received the B.S. incommunication engineering from Nanjing University of Science and Technology and M.S. in information science and engineering from Nanjing University of Technology, Nanjing, China in 2006 and 2009, respectively, Since 2009, he has been serving as a lecturer in Nantong Campus, Jiangsu Cit Vocational College. His research interests mainly include target localization, time delay estimation and signal processing.

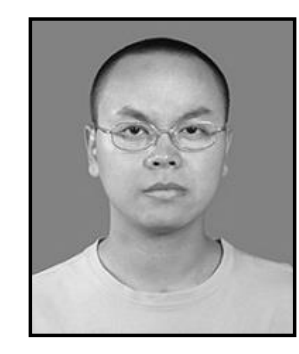

Yi Huang He, he received the B.S. and M.S. in information science and engineering School from Nanjing University of Technology, Nanjing, China in 2006 and 2009, respectively, and the $\mathrm{Ph} . \mathrm{D}$. degree in information and communication engineering from Southeast University (SEU), Nanjing, China, in 2014. Since 2014, he has been serving as a lecturer in Jiangsu University of Science and Technology (JUST). His research interests mainly include target localization, time delay estimation and signal processing. 